\title{
One Shot Dilatation (OSD) in Percutaneous Nephrolithotomy (PCNL) - A Safe and Feasible Technique
} Abdul Matin Anamur Rashid Choudhury ${ }^{1}$, Tohid Mohammad Saiful Hossain ${ }^{2}$, Tasmina Parveen ${ }^{3}$

Received: 16 - 08 - 2020

Accepted: 05 - 10 - 2020

Conflicts of interest: None

Keywords: Calyceal access, complications, dilatation, percutaneous nephrolithotomy, renal calcul, nephrostomy, feasibility.

\begin{abstract}
:
Objective: To evaluate the feasibility, safety and efficacy of one-shot dilation (OSD) in standard percutaneous nephrolithotomy (PCNL).
\end{abstract}

Methods: This is a prospective, single-center study conducted between January 2017 to June 2019. Patients who underwent PCNL using one shot dilatation (21 - 24 Fr) for access in PCNL were included in the study. Access time, fluoroscopy time, successful dilation time, stone free rate, decrease hemoglobin level and transfusion rate, complication rate, length of post operative hospital stay, cost were recorded. Data was analyzed and reported using summary statistics.

Results: A total of 30 patients were enrolled in this survey. 30 patients in one shot dilation (OSD) group. The mean age was 39.5 years. Control group of 30 patients were included in standard PCNL procedure where method of tract dilation was done by regid Amplatz dilators. Access time and fluoroscopy time in OSD group was approximately shorter. Statistically it was significant. Successful dilation and stone free rate were subsequently $98 \%$ and was significant. Data collected included patient access time, radiation exposure, total operating time, preoperative and postoperative hemoglobin concentrations, tract dilatation failures, complications and transfusions.

Conclusion: The use of one shot and standard prone position under general anaesthesia combines the advantages of these both methods including less radiation exposure and shorter access and operative time. One shot dilation technique is safe, economical and feasible technique with added advantage, lesser radiation exposure and lesser chance of blood transfusion.

\section{Introduction:}

Recent development of minimally invasive treatment of urinary calculi, percutaneous nephrolithotomy (PCNL) has become one of the main treatment for large kidney and upper ureteral stones. ${ }^{1}$ One of the most fundamental steps of PCNL surgery is to establish safe and effective access. However, complication in this process such as tract dilatation failure, hemorrhage and perforation of the renal parenchyma or collecting system are not uncommon. Consequently, it is especially important to identify a simple, effective and safe tract dilatation method for clinical application., ${ }^{2,3}$ Currently, the primary dilatation method of access creation in PCNL can be classified as one- shot dilatation (OSD) and serial dilatation. ${ }^{4}$

Currently, the evidence regarding the efficacy and safety of these two methods is still controversial. In recent years,

1. Associate Professor, Department of Urology, National Institute of Kidney diseases and Urology (NIKDU), Dhaka.

2. Associate Professor, Department of Urology, Bangabandhu Sheikh Mujib Medical University (BSMMU), Dhaka.

3. Professor \& Head, Department of Physiology, Uttara Adhunik Medical College, Dhaka.

Correspondence: Dr. Abdul Matin Anamur Rashid Choudhury, Associate Professor, Department of Urology, National Institute of Kidney diseases and Urology (NIKDU), Dhaka-1207, Bangladesh. E-mail: dr_choudhury2001@yahoo.com 
an increasing number of studies have shown that OSD is associated with more advantage than serial dilatation, which attracted much attention from urologists. 5,6

\section{Methods:}

This is a prospective, single -center study conducted between January 2017 to June 2019. Patients who underwent PCNL using single step dilatation (20- 24 Fr) for access in PCNL were included in the study.

\section{Inclusion criteria:}

- Patients of either sex

- $\quad$ Age ranged (31-48) years

- Renal calculus size $>2 \mathrm{~cm}$ or multiple renal calculi.

\section{Exclusion criteria:}

- Bleeding disorders

- Uncontrolled urinary tract infection

- Pregnant women

Patients of either sex, aged $>31$ years with renal calculus of size $>2 \mathrm{~cm}$ and / or multiple renal calculi who underwent PCNL with single -step dilatation at our tertiary care hospital were included in the study. The decision of PCNL was based on clinical judgement and was not prespecified by the study protocol. Patients with bleeding disorders, uncontrolled urinary tract infection or pregnant women were excluded from the study. The surgical procedure was carried out by a team of

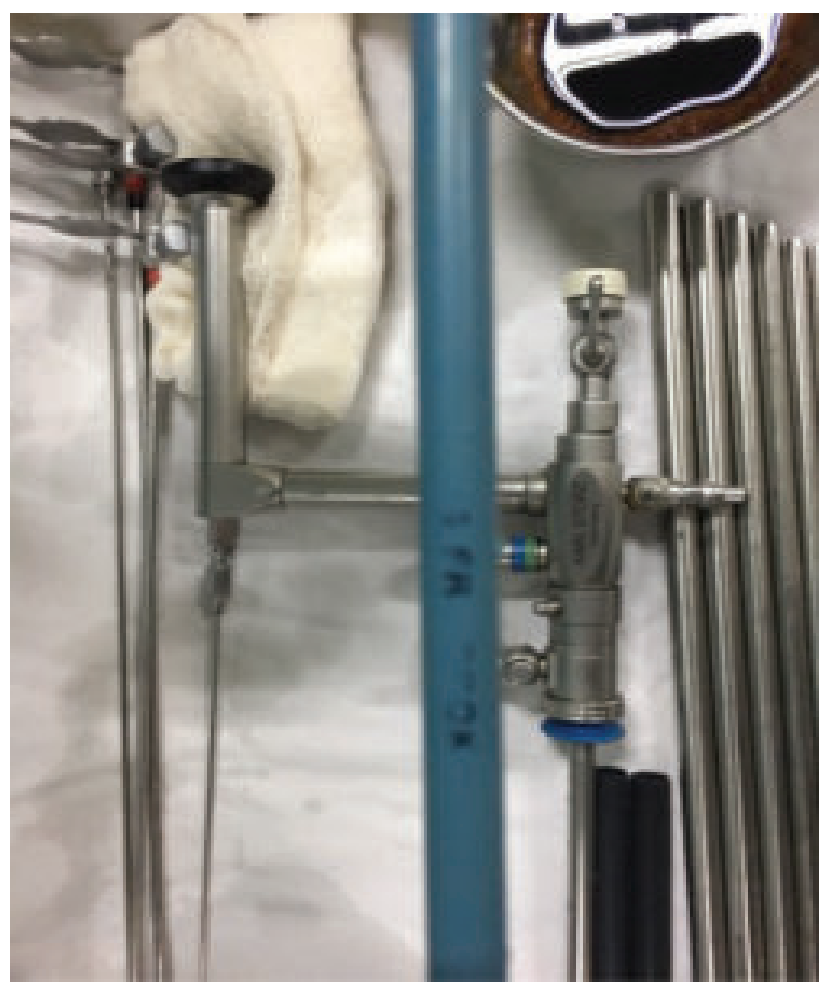

Fig.-1: One Shot Dilation set experienced urologists comprising two surgeons with similar experience and one senior urologists.

Standard surgical technique: Preoperatively, patient undergoes complete blood count, renal function test and coagulation profile. The PCNL is performed with patient in prone position under general anesthesia the patient is placed in lithotomy position, cystoscopy is done, and 5 Fr ureteric catheter inserted and fixed to transurethral Foleys catheter. The patient is then repositioned to prone position and pelvical system is delineated by retrograde injection of contrast material. The 18 G PCNL access needle is used to puncture. The desired calyx under fluoroscopic/ C-arun guidance and guide wire is passed into calyx/pelvis/ureter. An 8-10 Fr Alken needle dilator is used for initial dilation of tract for introduction of guide $\operatorname{rod}^{5,6}$.

Single-step dilatation: Single-step dilation was done by slowly advancing the Amplatz dilator (21-30 Fr) of desired size under C-arun guidance/ fluoroscopy, some diameter access sheath was glided over the dilator, similar technique was followed if patient required second or multiple punctures in a complex stone. A 20 Fr rigid nephroscope was used for the procedure and pneumatic lithotripter was used for fragmentation of stones. At the end of procedure DJ stent or a nephrostomy tube was inserted. 5,6,7

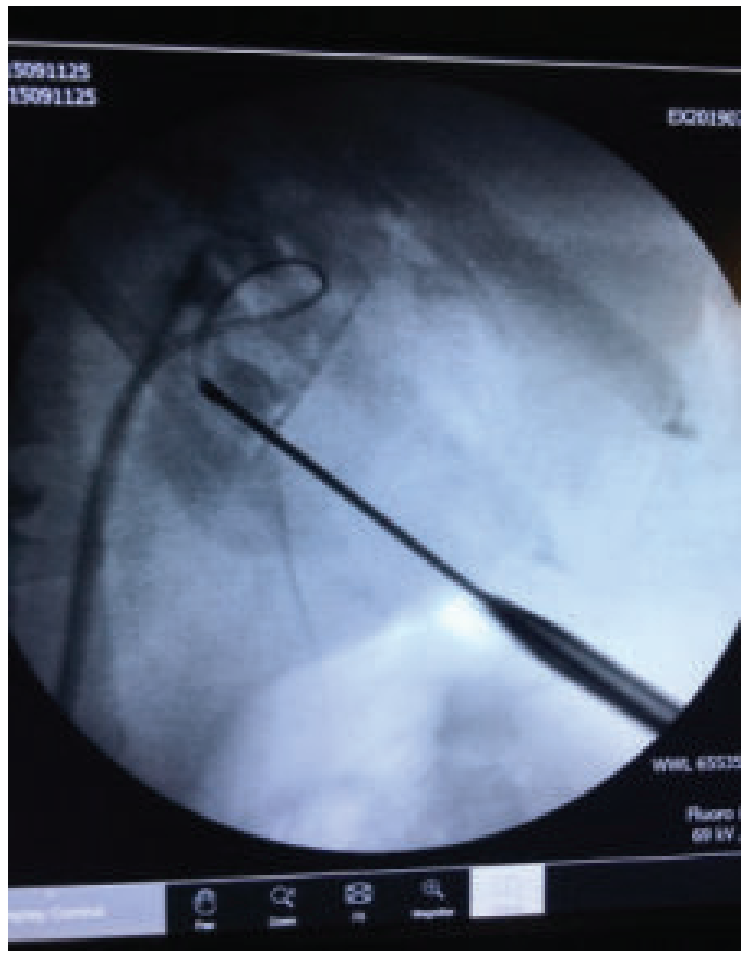

Fig.-2: The Single 24 Frdilator pulled in theAlken under fluoroscopic control 

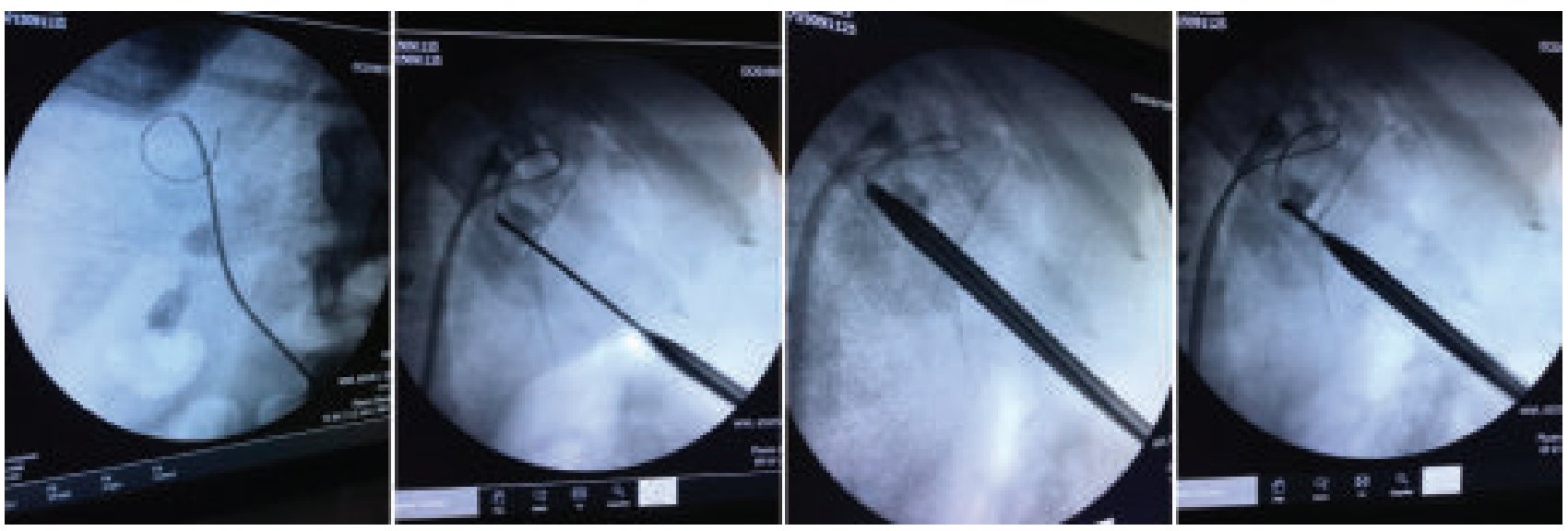

Fig.-3: Ongoing procedure of one shot dilation technique of PCNL

Results:

Table I : Summary Observations

\begin{tabular}{lccc}
\hline Parameter & $\begin{array}{c}\text { OSD (one shot } \\
\text { dilation) }(\mathrm{n}=30)\end{array}$ & $\begin{array}{c}\text { SD (serial } \\
\text { dilation) }(\mathrm{n}=30)\end{array}$ & P-value \\
\hline Access Time & $110 \mathrm{sec}$ & $118 \mathrm{sec}$ & $<0.001$ \\
Fluroscopy time & $10 \mathrm{~min}$ & $15 \mathrm{~min}$ & $<0.001$ \\
Successful dilation rate & $98 \%$ & $89 \%$ & $<0.001$ \\
Stone free rate & $98 \%$ & $88 \%$ & $<0.001$ \\
Decrease hemoglobin level and transfusion rate & $02 \%$ & $07 \%$ & $<0.05$ \\
Complication rate & $03 \%$ & $09 \%$ & $<0.001$ \\
Length of pos operative hospital stay & 3 days & 8 days & $<0.001$ \\
Cost & less & More & $<0.05$ \\
\hline
\end{tabular}

Overall,30 patients were enrolled in OSD technique. The mean (SD) age was 39.5 years and ranged from 31-48 years. Among these 22 were male and 08 were female.

In table $\mathrm{I}$ it is observed that Access time and fluoroscopy time in OSD group was approximately shorter. Statistically it was significant. Successful dilation and stone free rate were subsequently $98 \%$ and were significant. The hemoglobin level decreased less in the OSD group.Complication was less in OSD group and post operative hospital staywas lower in OSD group. Overall cost of operation was less in OSD group.

\section{Discussion:}

PCNL is the main treatment method for large and complex kidney stones. The creation of a nephrostomy tract is one of the most basic steps of PCNL. ${ }^{7,8}$ Significant reduction in access time and fluoroscopy time with OSD .The successful dilation rate or transfusion rate was maximum, $98 \%$. In addition, no difference was observed in the stone-free rate or complication rate. However, postoperative haemoglobin levels decreased less with OSD.9-12

OSD involving a single dilation of the tract with a (2124) Fr dilator is simple and does not require gradual tract dilation. ${ }^{13}$ It saves access time and X-ray exposure during tract creation, thus reducing the operative time and decreasing the risk of radiation damage to patients and operators. In addition, the simple surgical procedures allow surgeons to easily master the technique ${ }^{12}$. In our experience, successful dilation and stone-free rates are important factors that influence the effectiveness of tract dilation techniques. ${ }^{14,15}$ According to our analysis, the successful dilation rate and stonefree rate of the OSD group were slightly lower. Factors that influence the successful dilation rate may be related to the patient's body mass index, a history of kidney surgery and the surgeon's experience. ${ }^{12,13}$ 
The postoperative haemoglobin levels decreased significantly less in patients who underwent OSD.The investigators found that the amount of intraoperative blood loss caused by the tract dilation technique accounted for half of the total blood loss. The correct puncture path and appropriate tract dilation methods were key decisive factors that determined the amount of intraoperative blood loss. The OSD method may effectively lessen postoperative decreases in haemoglobin levels by reducing the amount of bleeding during surgery. ${ }^{15}$

In this study, the overall complication rate was $12 \%$ in all patients $(0.3 \%$ in the OSD group and $09 \%$ in the serial dilation group). The main complication included postoperative urinary tract infections, urine leakage, haemorrhage, haematoma formation and postoperative fever. The difference in complication rates was not statistically significant between the OSD group and the serial dilation group.

\section{Conclusion:}

Percutaneous access can be safely and successfully obtained by one shot dilation even in standard prone position. The use of this dilation technique in standard prone position. Combines their both advantages including less radiation exposure and shorter access and operative time. The one shot dilation is also cost effective, easy to learn and offers a potential alternative to the standard devices particularly in developing countries. Further analysis and comparative studies are necessary to confirm these results. The results of this study suggest that the OSD is a safe and efficacious tract dilation technique that can reduce the access time, fluoroscopy time and postoperative decreases in haemoglobin levels. The length of hospital stay is less.

\section{References:}

1. Suelozgen T, Isoglu CS, Turk H, Yoldas M, Karabicak M, Ergani B, et al. Can we use singlestep dilation as a safe alternative dilation method in percutaneous nephrolithotomy? Urology. 2017; 99:38-41.

2. Kalpee AR, Venter R, Fourie T. Single-centre comparison of a novel single-step balloon inflation device and amplatz sheath dilatation during percutaneous nephrolithotomy: A pilot study. S Afr J Surg. 2012; 50:79-81.

3. 3.Nour HH, Kamal AM, Zayed AS, et al. Singlestep renal dilatation in percutaneous nephrolithotomy: A prospective randomised study. Arab J Urol2014; 12:219-22.
4. Aminsharifi A, Alavi M, Sadeghi G, et al. Renal parenchymal damage after percutaneous nephrolithotomy with one-stage tract dilation technique: a randomized clinical trial. J Endourol2011; 25:927-31.

5. Falahatkar S, Neiroomand H, Akbarpour M, et al. One-shot versus metal telescopic dilation technique for tract creation in percutaneous nephrolithotomy: comparison of safety and efficacy. J Endourol2009; 23:615-8.

6. Amjadi M, Zolfaghari A, Elahian A, et al. Percutaneous nephrolithotomy in patients with previous open nephrolithotomy: one-shot versus telescopic technique for tract dilatation. J Endourol2008; 22:423-6.

7. Fernström I, Johansson B. Percutaneous pyelolithotomy. A new extraction technique. Scand J UrolNephrol. 1976; 10:257-9.

8. Bai Y, Tang Y, Deng L, Wang X, Yang Y, Wang J, et al. Management of large renal stones: Laparoscopic pyelolithotomy versus percutaneous nephrolithotomy. BMC Urol. 2017; 17:75.

9. Ganpule AP, Vijayakumar M, Malpani A, Desai MR. Percutaneous nephrolithotomy (PCNL) a critical review. Int J Surg. 2016; 36:660-4.

10. El-Shazly M, Salem S, Allam A, Hathout B. Balloon dilator versus telescopic metal dilators for tract dilatation during percutaneous nephrolithotomy for staghorn stones and calyceal stones. Arab J Urol. 2015; 13:80-3.

11. Nour HH, Kamal AM, Zayed AS, Refaat H, Badawy MH, El-Leithy TR, et al. Single-step renal dilatation in percutaneous nephrolithotomy: A prospective randomised study. Arab J Urol. 2014; 12:219-22.

12. Frattini A, Barbieri A, Salsi $P$, et al. One shot: a novel method to dilate the nephrostomy access for percutaneous lithotripsy. J Endourol2001; 15:919-23.

13. Dehong C, Liangren L, Huawei L, et al. A comparison among four tract dilation methods of percutaneous nephrolithotomy: a systematic review and meta-analysis. Urolithiasis 2013; 41:523-30.

14. Li Y, Yang L, Xu P, et al. One-shot versus gradual dilation technique for tract creation in percutaneous nephrolithotomy: a systematic review and meta-analysis. Urolithiasis 2013; 41:443-8.

15. Kessaris DN, Bellman GC, Pardalidis NP, et al. Management of hemorrhage after percutaneous renal surgery. J Urol 1995; 153(3 Pt 1):604-8. 